\title{
High Amounts of S100-Alarmins Confer Antimicrobial Activity on Human Breast Milk Targeting Pathogens Relevant in Neonatal Sepsis
}

\begin{abstract}
Sabine Pirr ${ }^{1 * t}$, Manuela Richter ${ }^{1,2+}$, Beate Fehlhaber ${ }^{1}$, Julia Pagel ${ }^{3}$, Christoph Härtel ${ }^{3}$, Johannes Roth ${ }^{4}$, Thomas Vogl ${ }^{4}$ and Dorothee Viemann ${ }^{1}$

'Department of Pediatric Pneumology, Allergy and Neonatology, Hannover Medical School, Hannover, Germany, ${ }^{2}$ Children's Hospital "Auf der Bult", Hannover, Germany, ${ }^{3}$ Department of Pediatrics, University of Lübeck, Lübeck, Germany, ${ }^{4}$ Institute of Immunology, University of Münster, Münster, Germany
\end{abstract}

Sepsis is a leading cause of perinatal mortality worldwide. Breast milk (BM) feeding is protective against neonatal sepsis, but the molecular mechanisms remain unexplained. Despite various supplementations with potential bioactive components from BM formula feeding cannot protect from sepsis. S100-alarmins are important immunoregulators in newborns preventing excessive inflammation. At high concentrations, the S100A8/A9 protein complex also has antimicrobial properties due to its metal ion chelation capacity. To assess whether BM contains S100-alarmins that might mediate the sepsis-protective effect of BM 97 human BM samples stratified for gestational age, mode of delivery and sampling after birth were collected and analyzed. S100A8/A9 levels were massively elevated after birth $(p<0.0005)$. They slowly decreased during the first month of life, then reaching levels comparable to normal values in adult serum. The concentration of S100A8/A9 in BM was significantly higher after term compared with preterm birth (extremely preterm, $p<0.005$; moderate preterm, $p<0.05$ ) and after vaginal delivery compared with cesarean section $(p<0.0005)$. In newborn s100a9-/- mice, enterally supplied S100-alarmins could be retrieved systemically in the plasma. To explore the antimicrobial activity against common causal pathogens of neonatal sepsis, purified S100-alarmins and unmodified as well as S100A8/A9-depleted BM were used in growth inhibition tests. The high amount of S100A8/A9 proved to be an important mediator of the antimicrobial activity of BM, especially inhibiting the growth of manganese $(\mathrm{Mn})$ sensitive bacteria such as Staphylococcus aureus $(p<0.00005)$ and group B streptococci $(p<0.005)$. Depletion of S100A8/A9 significantly reduced this effect $(p<0.05$, respectively). The growth of Escherichia coli was also inhibited by BM $(\rho<0.00005)$ as well as by S100A8/A9 in culture assays $(p<0.05)$. But its growth in BM remained unaffected by the removal of S100A8/A9 and was neither dependent on Mn suggesting that the antimicrobial effects of S100A8/A9 in BM are primarily mediated by its Mn chelating capacity. In summary, the enteral supply of bioavailable, antimicrobially active amounts of S100-alarmins might be a promising option to protect newborns at high risk from infections and sepsis.

Keywords: neonate, sepsis, S100A8/A9, alarmins, breast milk 


\section{INTRODUCTION}

Breastfeeding and human milk represent the gold standard for infant feeding and nutrition (1). Clinical and epidemiological studies indicate the short- and long-term medical and developmental advantages of breastfeeding compared with formula feeding, i.e., lower incidence of infectious and inflammatory diseases, reduction of the risk for asthma, allergy, and obesity as well as promotion of gastrointestinal, immune, and neurologic development (1-3). Although immunoregulatory, antimicrobial, and antiviral activities have been linked to diverse bioactive components $(2,4,5)$, our knowledge on the molecular mechanisms that confer these properties on breast milk (BM) are still very fragmentary.

Newborn infants, especially preterm babies, are at high risk for fatal sepsis characterized by rapid courses with hyperinflammatory immune responses (6). Sepsis is still a leading cause of death among neonates worldwide (7-9). BM feeding significantly decreases the incidence of sepsis in newborns (10-12). However, most efforts of nutritional supplementation of formula with bioactive components from human BM remained without success with respect to decreasing the incidence of infection and inflammation $(2,13)$.

We previously showed that the endogenous toll-like receptor 4 ligands S100A8 and S100A9 (also known as MRP8 and MRP14 or calprotectin) have an important role in newborns by regulating inflammatory responses (14-16). Activated myeloid cells release heterodimeric complexes of S100A8 and S100A9 called calprotectin (S100A8/A9) (17). Reduced release of these S100-alarmins at birth is associated with an increased risk of newborns to suffer from sepsis (15). Systemic application of S100-alarmins protects neonatal s100a $9^{-/-}$mice from hyperinflammation and fatal courses of sepsis $(15,16)$. Next to these immunoregulatory effects on innate immune responses, inhibiting as well as promoting effects on the growth of bacteria have been ascribed to S100A8/ A9 (18-21). Growth inhibition of bacteria by S100A8/A9 has been shown for Staphylococcus aureus and Klebsiella pneumoniae. This effect has been attributed to the metal ion chelation capacity of S100A8/A9 that becomes relevant at high concentrations of S100A8/A9 starting at $20 \mu \mathrm{g} / \mathrm{mL}(18,19)$.

In this study, we aimed at analyzing whether human BM contains S100-alarmins that contribute to the sepsis-protective effect of BM by providing direct antibacterial effects on pathogens relevant in neonatal sepsis. Accordingly, human BM samples stratified for gestational age, mode of delivery, and sampling after birth were collected at tertiary neonatal care hospitals to determine S100A8/A9 levels and the antimicrobial activity that can be attributed to these proteins.

\section{MATERIALS AND METHODS}

\section{Study Approval}

The studies were approved by the Institutional Review Board of Hannover Medical School (no. 6143-2012). Mouse experiments

Abbreviations: BM, breast milk; CFU, colony-forming units; CS, cesarian section; E. coli, Escherichia coli; GBS, group B streptococci; IP, immunoprecipitation; S. aureus, Staphylococcus aureus; TLR4, toll-like receptor 4; VD, vaginal delivery. were in accordance with German Animal Welfare Legislation and performed as approved by the Lower Saxony State Office for Consumer and Food Safety, Germany (approval no. 33.12-4250204-14 and no. 33.12-42502-04-15).

\section{Study Participants}

Human BM samples $(n=97)$ were collected from mothers who gave birth to a live baby of 22-41 gestational weeks in the Departments of Obstetrics at the Hannover Medical School, the Henriettenstift Hannover, or the University of Lübeck (Table 1). Written informed consent was obtained from all participating women. Women with chorioamnionitis or systemic infections, major inborn abnormalities, inborn errors of metabolism, and immune-related illnesses were excluded. Mothers were assigned to three groups according to the gestational age of their baby: $<32$ weeks (extremely and very preterm) ( $n=41), 32-36$ weeks (moderate preterm) $(n=37)$, and $\geq 37$ weeks (term) $(n=21)$. Gestational age was calculated based on the last menstrual period. When early ultrasound at $11-13^{+6}$ weeks' gestation using the fetal crown-rump length deviated more than 7 days, dating was performed using ultrasound. Milk was collected on days $1-2(n=12), 3-5(n=19)$, $6-10(n=35), 11-14(n=8), 15-30(n=13)$, and $>30(n=10)$ after birth. Milk was expressed by mothers according to their individual feeding protocol either manually or by using a breast pump. Two milliliters of expressed milk were stored at $4^{\circ} \mathrm{C}$ for a maximum of $12 \mathrm{~h}$. After centrifugation at $1,200 \times \mathrm{g}$ for $10 \mathrm{~min}$ supernatants were stored at $-80^{\circ} \mathrm{C}$ until analyzed for S100A8/ A9 concentrations.

\section{Reagents}

The human S100A8/A9 heterodimer (hS100A8/A9) was isolated from granulocytes as described earlier (22). Human S100A8 and S100A9 were recombinantly expressed in Escherichia coli BL21(DE3) as described previously $(14,23)$. Proteins were analyzed by amino acid sequencing and electrospray ionization mass spectrometry. All preparations revealed $>98 \%$ purity. Endotoxin contaminations were excluded by limulus amebocyte lysate assay (BioWhittaker), heat inactivation $\left(30 \mathrm{~min}\right.$ at $\left.80^{\circ} \mathrm{C}\right)$, and polymyxin-B-blocking experiments.

\section{ELISA Assay}

Concentrations of S100A8/A9 in human BM were determined by an in-house ELISA as described previously $(14,24)$.

\section{Mice}

s100a $9^{-/-}$mice (25) were used for breeding under specific pathogen-free conditions at the Central Animal Facility at the University of Veterinary Medicine Hannover. Enteral administration of S100-alarmins was performed at the age of 1 day by gastric tube feeding. $s 100 a 9^{-/-}$pups received $20 \mu \mathrm{g} \mathrm{hS100 \textrm {A } 8 /}$ A9 complex or $5 \mu \mathrm{g}$ hS100A9 in $20 \mu \mathrm{L}$ of phosphate-buffered saline (PBS) or aqua alone [control (Ctrl)]. Intraperitoneal injection of hS100A9 and PBS served as positive and negative Ctrls. Plasma was collected $24 \mathrm{~h}$ after application of the S100 proteins and immunoblotted against S100A9. 
TABLE 1 | Birth-associated characteristics of the study group providing breast milk samples

\begin{tabular}{|c|c|c|c|c|c|}
\hline Gestational age & All & $<32$ weeks & $32-36$ weeks & $\geq 37$ weeks & $p$-Value \\
\hline Sample size $n(\%)$ & $97(100)$ & $40(41)$ & $37(38)$ & $20(21)$ & \\
\hline Mean gestational age (SD) & $33.0(4.6)$ & $28.6(2.4)$ & $34.0(1.2)$ & $39.6(1.5)$ & $<0.001^{a}$ \\
\hline Mean age of mothers (SD) & $32.3(6.7)$ & $32.8(7.3)$ & $32.3(6.0)$ & $31.1(6.7)$ & $0.639^{a}$ \\
\hline Mean parity (SD) & $1.7(1.2)$ & $1.5(0.8)$ & $1.6(0.7)$ & $2.2(2.4)$ & $0.108^{a}$ \\
\hline Vaginal delivery $n(\%)$ & $33(34)$ & $11(27)$ & $11(30)$ & $11(55)$ & \multirow{2}{*}{$0.090^{b}$} \\
\hline Cesarean section $n(\%)$ & $64(66)$ & $29(73)$ & $26(70)$ & $9(45)$ & \\
\hline
\end{tabular}

aOne-way ANOVA, comparison of all three gestational groups.

${ }^{b}$ Fisher's exact test, comparison of the proportional distribution of delivery modes between all three gestational groups.
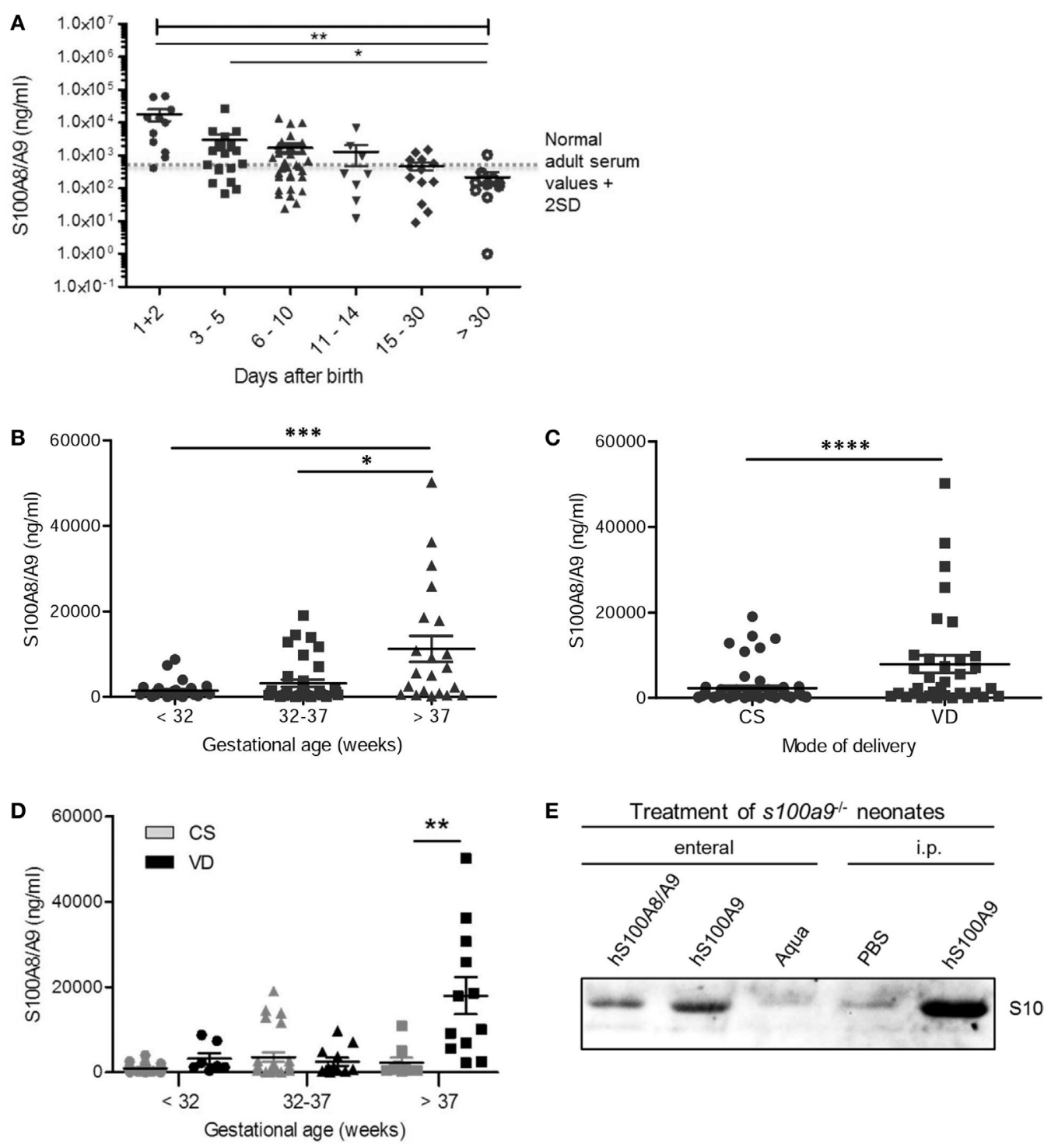

$\mathbf{E}$

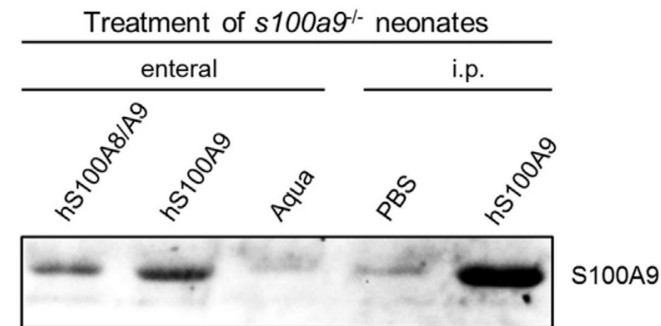

FIGURE 1 | Levels and bioavailability of S100A8/A9 in human breast milk (BM). (A) Postnatal course of S100A8/A9 levels in human BM. Bars on a logarithmic scale represent means \pm SEM. Significant differences are indicated by capped-end lines across groups $\left(p<0.0005\right.$, Kruskal-Wallis test) and open lines $\left({ }^{*} p<0.05\right.$ and ${ }^{\star \star} p<0.0005$, post hoc Mann-Whitney U-test). (B-D) Mean S100A8/A9 levels in human BM during the first 30 days were categorized according to the baby's gestational age (B), the mode of delivery (C), and the mode of delivery within the different gestational age groups (D). Bars represent means \pm SEM. ${ }^{\star} p<0.05$, ${ }^{\star \star} p<0.01,{ }^{\star \star \star} p<0.005$, and ${ }^{\star \star \star \star} p<0.0005$, $t$-test. CS, Cesarean section; VD, vaginal delivery. (E) S100A9 immunoblotting of plasma from s100a9-/- neonates collected $24 \mathrm{~h}$ after enteral feeding of hS100A8/A9, hS100A9, or aqua. Intraperitoneal (i.p.) injection of hS100A9 and phosphate-buffered saline served as positive and negative control. 


\section{Bacterial Strains, Media, Growth Conditions}

Todd-Hewitt-Bouillon (Roth, Karlsruhe, Germany) was inoculated 1:100 with overnight cultures of $S$. aureus strain Newman (GenBank accession number AP009351.1), E. coli (ATCC 25922), and group B Streptococcus agalactiae [group B streptococci (GBS), WT 199] and incubated at $37^{\circ} \mathrm{C}$ with constant shaking at $200 \mathrm{rpm}$ until late-logarithmic growth phase was reached according to optical density at $600 \mathrm{~nm}\left(\mathrm{OD}_{600}\right)$, respectively. Bacterial suspensions were concentrated 50 times in succeeding centrifugation steps until the final pellet was resuspended in PBS at a concentration of about $1 \times 10^{10}$ colonyforming units $(\mathrm{CFU}) / \mathrm{mL}$.

\section{Bacterial Growth Inhibition Assays}

To test direct antimicrobial activity, S100A8/A9 and S100A8 were diluted in HBSS [without calcium (Ca) and magnesium] at $100 \mu \mathrm{g} / \mathrm{mL}$ while bacterial suspensions of S. aureus, E. coli and GBS were diluted 10-fold three times in RPMI medium (Biochrom AG, Berlin, Germany) with $25 \mathrm{mM}$ HEPES. Equal volumes of both preparations were mixed and $200 \mu \mathrm{L}$ culture aliquots grown at $37^{\circ} \mathrm{C}$ while shaking at $180 \mathrm{rpm}$ in a 96-well microtiter plate. Bacterial suspensions mixed with HBSS without S100A8 or S100A8/A9 served as Ctrls. Aliquots of the cultures were taken after $0,2,5,8$, and $24 \mathrm{~h}$, diluted and plated onto agar plates to determine the number of CFU, respectively. Bacterial growth in the presence of different concentrations of S100A8/A9 with and without the addition of $0.5 \mathrm{mM} \mathrm{Ca}$ or in the presence of different concentrations of EDTA and manganese (Mn) was monitored by measuring the increase in $\mathrm{OD}_{600}$ over time.

To determine the antimicrobial activity of formula (Humana 0 ), BM and S100A8/A9-depleted BM, equal volumes were mixed with bacterial suspensions to receive a final concentration of $1 \times 10^{6} \mathrm{CFU} / \mathrm{mL}$. Before usage, milk samples were fat reduced by centrifugation at $6,000 \times g$ for $10 \mathrm{~min}$. Aliquots of $200 \mu \mathrm{L}$ from the bacteria-milk suspensions were grown at $37^{\circ} \mathrm{C}$ while shaking at $180 \mathrm{rpm}$ in a 96 -well microtiter plate. Aliquots of the cultures were taken after 0 and $24 \mathrm{~h}$, diluted and plated onto agar plates to determine the number of CFU, respectively.

\section{Immunoprecipitation (IP) of S100A8/A9 from BM}

For the removal of S100A8/A9, BM was first centrifuged at $500 \times g$ for $25 \mathrm{~min}$ and again at $5,000 \times g$ for $30 \mathrm{~min}$. The skimmed supernatant was precleared two times for $1.5 \mathrm{~h}$ with Protein A/G Agarose beads (Thermo Scientific, Rockford, IL, USA). Subsequently, the BM was incubated overnight with beads coupled either with the monoclonal antihuman S100A8/A9 antibody (clone 27E10 purified by Vogl) (S100-IP) or a nonspecific polyclonal goat anti-mouse Ctrl antibody (goat antimouse IgG-HRP, sc-2005, Santa Cruz Biotechnology, Dallas, TX, USA) (Ctrl-IP). Probes from BM before and after S100-IP and Ctrl-IP as well as from the precipitates were retained for bacterial growth inhibition assays as well as immunoblotting against S100A8.

\section{Immunoblotting}

SDS-PAGE and Western blot staining was performed as described earlier $(14,15,26)$ using the primary polyclonal antihuman S100A8 and S100A9 antibody purified by Vogl (14). The antirabbit horseradish peroxidase conjugated secondary antibody was obtained from Cell Signaling (Leiden, Netherlands). Protein bands were visualized using the enhanced chemiluminescence system and the ChemiDoc MP System with Image Lab Software v. 4.0 (Bio-Rad Laboratories, Munich, Germany).

\section{Statistical Analysis}

Comparison of S100A8/A9 BM levels between defined groups was performed applying the Mann-Whitney $U$-test. Statistical

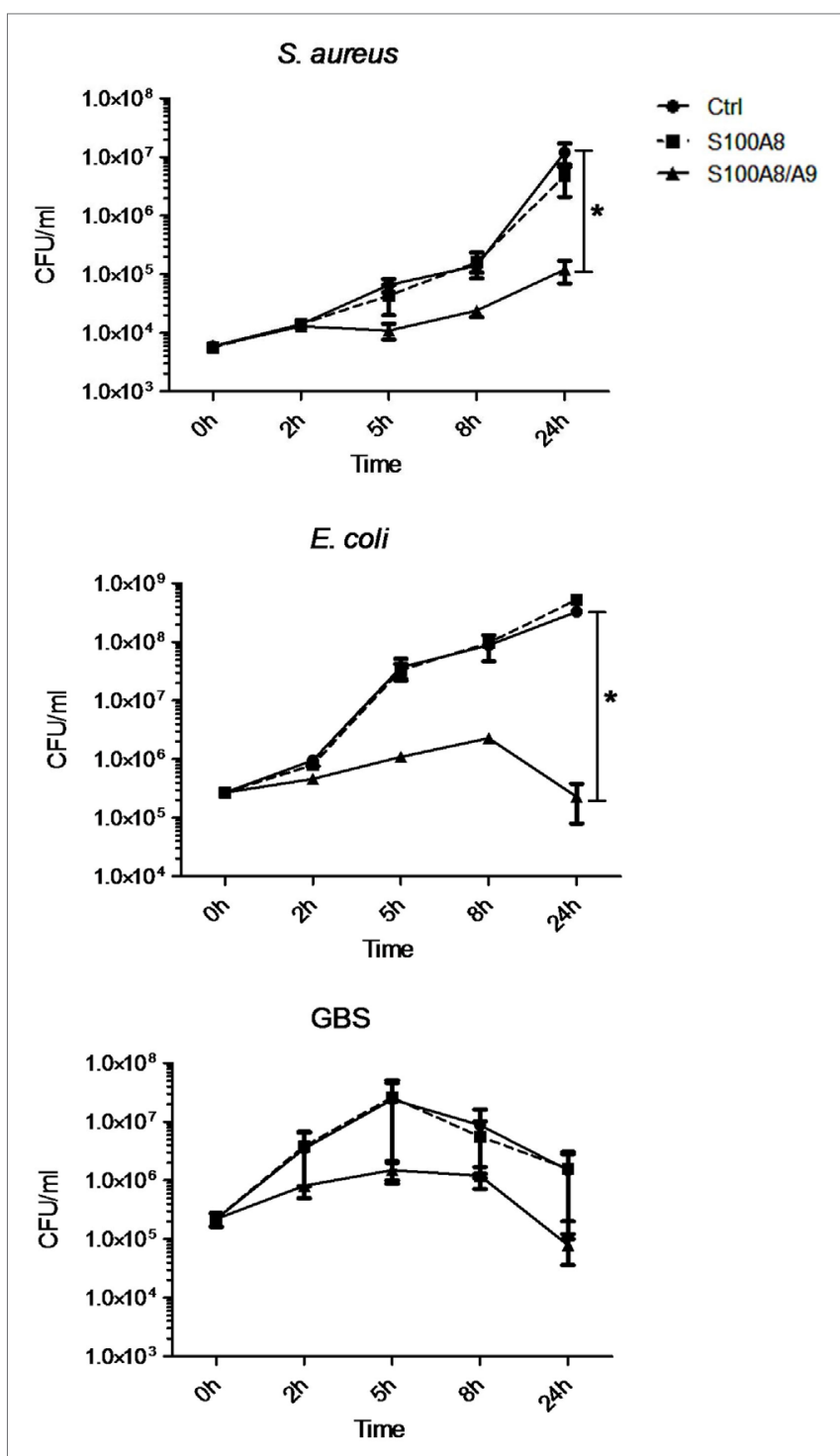

FIGURE 2 | S100A8/A9 inhibits the growth of pathogens causing neonatal sepsis. Staphylococcus aureus, Escherichia coli, and group B streptococci (GBS) were grown in the absence [control (Ctrl)] and presence of $50 \mu \mathrm{g} / \mathrm{mL}$ S100A8/A9 or S100A8. The concentration of colony-forming units (CFU) over culture time was plotted as mean \pm SEM. ${ }^{*} p<0.05$, $t$-test $(n=4)$. 
significance of S100A8/A9 BM levels changing over the time after birth were evaluated by running a Kruskal-Wallis test followed by a post hoc Mann-Whitney $U$-test. Comparison of bacterial growth kinetics at defined time points was performed by applying the unpaired two-tailed Student's $t$-test.

\section{RESULTS}

\section{S100A8/A9 Levels in BM Are Massively Elevated after Birth}

Ninety-seven samples of human BM were analyzed and stratified for the day of sampling after birth. During the first 2 days after birth, S100A8/A9 levels in BM were massively elevated $(18,400 \pm 6,500 \mathrm{ng} / \mathrm{mL})$ compared with normal serum levels in adults $(220 \pm 40 \mathrm{ng} / \mathrm{mL})$ determined previously (14) (Figure 1A). The levels gradually decreased over time, in average reaching normal adult serum levels 1 month after birth (Figure 1A). S100A8/A9 levels in BM of mothers who gave birth to term babies were significantly higher than of mothers with premature born infants (Figure 1B). Moreover, levels were significantly higher after vaginal delivery (VD) compared with delivery by cesarean section (CS) (Figure 1C). When regarding the gestational groups separately, significant dependence on the mode of delivery was only evident after term delivery. Within both preterm groups, the differences between VD and CS were not significant (Figure 1D).

To provide first evidence that enterally supplied S100-alarmins are bioavailable for the newborn, we used $s 100 a 9^{-/-}$mice that were fed with $20 \mu \mathrm{g}$ S100A8/A9 or $5 \mu \mathrm{g}$ S100A9 on day 1 of life. Twenty-four hours after feeding, the alarmins could be retrieved systemically in the plasma of these mice (Figure 1E). Collectively, the findings demonstrated that BM supplies newborn infants with high amounts of bioavailable S100-alarmins after birth, especially, when they are born at term and delivered vaginally.

\section{S100A8/A9 Acts Bacteriostatic against Important Pathogens of Neonatal Sepsis}

To clarify the antimicrobial role of $\mathrm{S} 100$ proteins in BM, we assessed the activity of S100A8/A9 and S100A8 on the growth of three of the most common pathogens causing neonatal sepsis. The heterodimer complex S100A8/A9 dose-dependently inhibited the growth of $S$. aureus, E. coli, and GBS (Figures 2 and 3), while the homodimer S100A8 exerted no antimicrobial activity (Figure 2), which was in accordance with previous reports (18). In the absence of $\mathrm{Ca}$, bacteriostatic activity against $S$. aureus and GBS required the presence of high concentrations of S100A8/A9 $(50 \mu \mathrm{g} / \mathrm{mL})$ (Figure 3A). However, in the presence of Ca, which is present in high amounts in $\mathrm{BM}$, the growth inhibitory activity of S100A8/A9 was significantly enhanced; then S100A8/A9 concentrations of $10 \mu \mathrm{g} / \mathrm{mL}$ against $S$. aureus and $20 \mu \mathrm{g} / \mathrm{mL}$ against GBS were sufficient for significant growth inhibition (Figure 3B). Impairment of $E$. coli growth also started at concentrations of $10-20 \mu \mathrm{g} / \mathrm{mL}$ S100A8/A9, but was independent of the presence of $\mathrm{Ca}$ (Figures 3A,B).

\section{S100A8/A9 Contributes to the Antimicrobial Activity of BM Targeting Mn-Sensitive Pathogens}

In line with previous reports of BM effects on $S$. aureus and E. coli $(27,28)$, we found the growth of S. aureus, E. coli, and

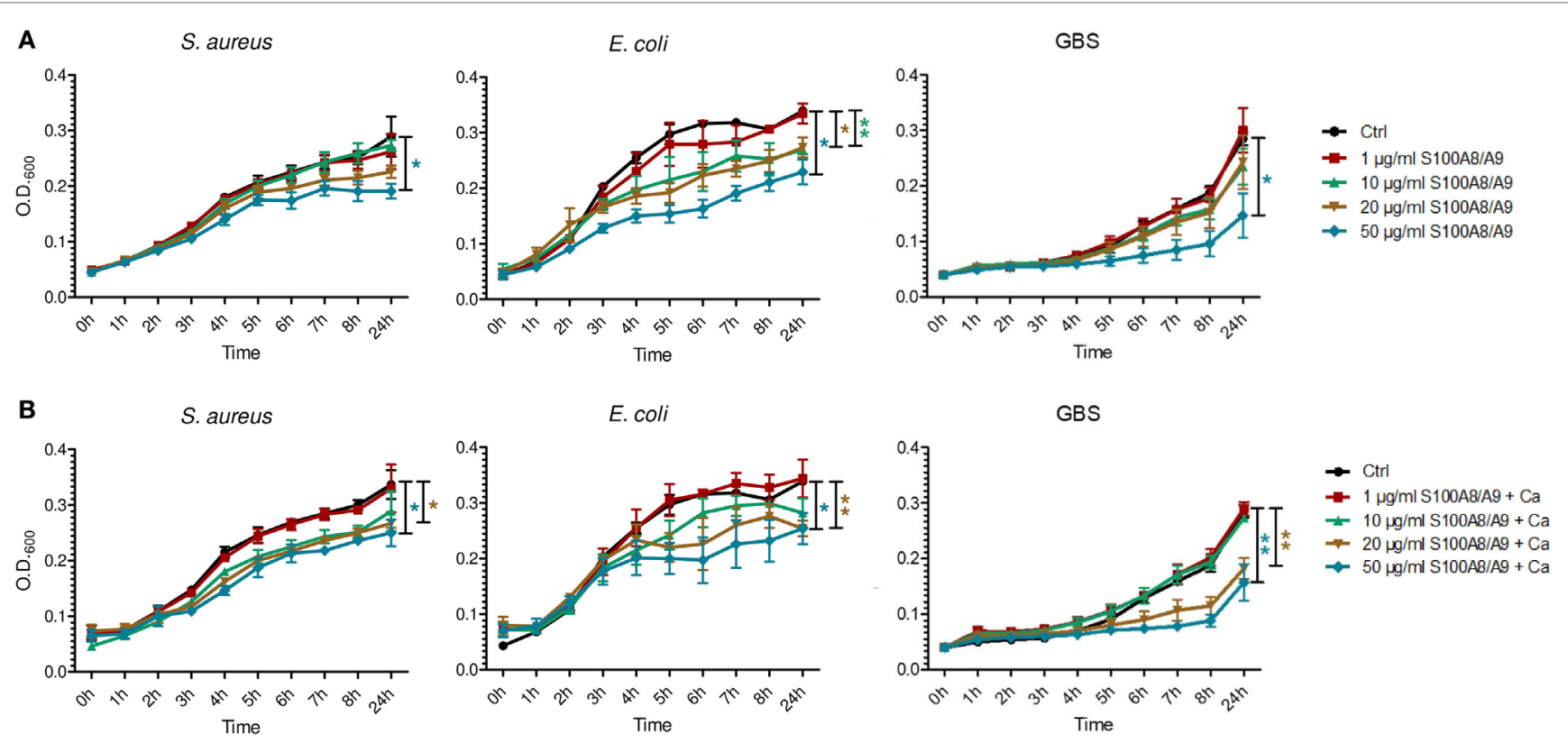

FIGURE 3 | S100A8/A9 exerts antimicrobial activity at concentrations detectable in breast milk. Staphylococcus aureus, Escherichia coli, and group B streptococci (GBS) were grown at indicated concentrations of S100A8/A9 in the absence (A) and presence of 0.5 mM Ca (B). OD 600 values over time were plotted as mean \pm SEM. ${ }^{*} p<0.05$ and ${ }^{* *} p<0.05$, $t$-test $(n=3)$ 
also GBS in BM significantly reduced compared with their growth in formula (Figure 4). To investigate whether and to what extent the presence of S100A8/A9 in BM is responsible for these effects we first elucidated what bacteriostatic mechanism of S100A8/A9 might be relevant in BM and for which of the bacterial strains. Chelation of divalent metal ions like Mn, zinc and iron has been reported to be the main mechanism of antimicrobial activity of S100A8/A9 $(18,19,29,30)$. Therefore, we first assessed the general susceptibility of the bacterial strains to metal ion depletion. The growth of $S$. aureus and GBS, but not of E. coli, was strongly impaired in the presence of EDTA (Figure 5A). Considering the molar ratios and binding
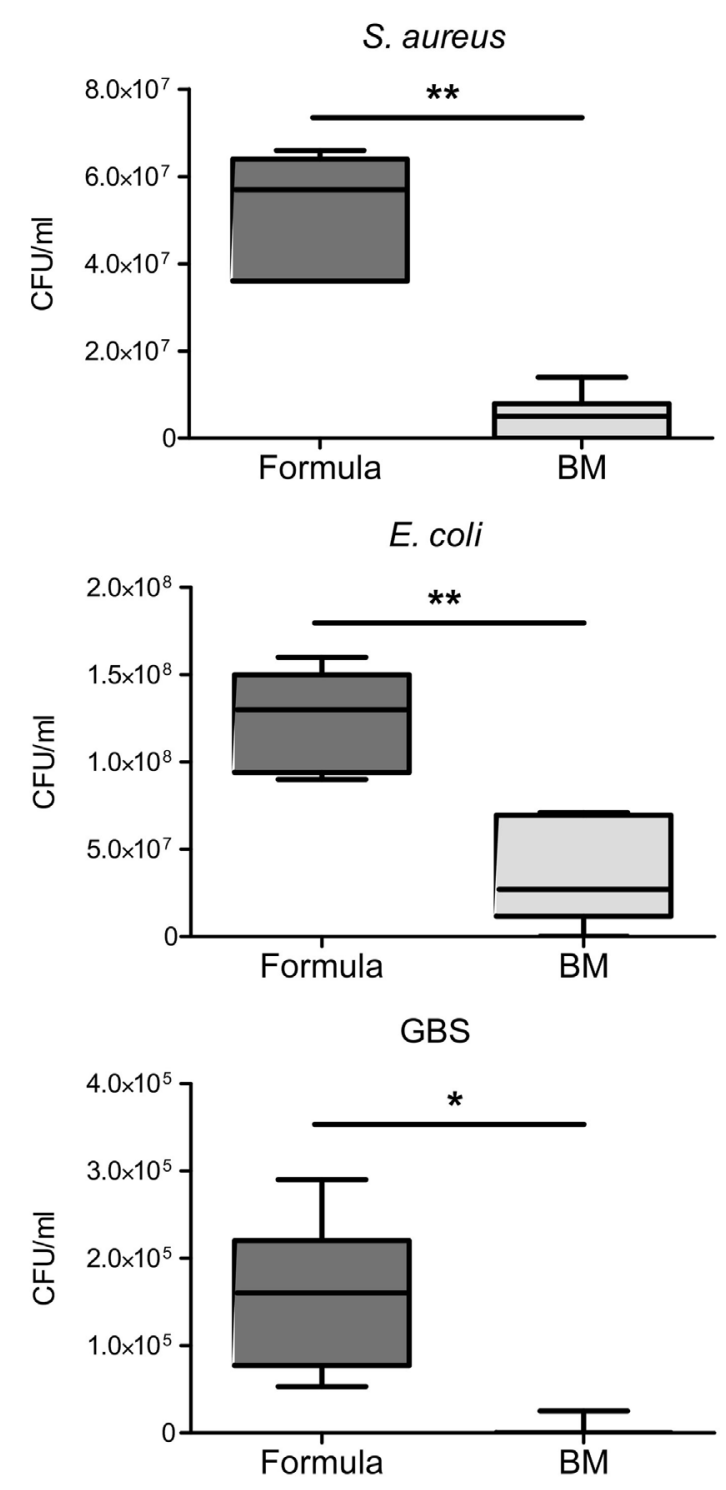

FIGURE 4 | Breast milk (BM) inhibits the growth of sepsis-causing pathogens. Staphylococcus aureus, Escherichia coli, and group B streptococci (GBS) were grown over $24 \mathrm{~h}$ in infant formula and BM. Box plots show medians (center lines) and interquartile ranges of the concentration of colony-forming units $(\mathrm{CFU}) \pm \mathrm{SEM} .{ }^{*} p<0.005$ and ${ }^{* *} p<0.00005, t$-test. capacities of S100A8/A9 for divalent ions, Mn depletion is the most likely antimicrobial mechanism of S100A8/A9 relevant in BM. A concentration of $50 \mu \mathrm{g} / \mathrm{mL}$ S100A8/A9 corresponds to $1 \mu \mathrm{M}$ S100A8/A9. Comparatively, zinc and iron are in excess in BM (about 0.1-0.3 mM). However, the average content of Mn in $\mathrm{BM}$ is only about $2 \mu \mathrm{M}$. Therefore, we tested the sensitivity of bacterial growth toward Mn supplementation. In contrast to E. coli, the growth of $S$. aureus and GBS was strongly promoted by $\mathrm{Mn}$ (Figure 5B), which was in line with the resistance of E. coli against divalent ion depletion by EDTA (Figure 5A). The data suggested that S100A8/A9-mediated antimicrobial activity of BM primarily might be relevant for $\mathrm{Mn}$-sensitive strains like S. aureus and GBS, but less important for largely Mn-insensitive bacteria like E. coli.

To validate this hypothesis, we removed S100A8/A9 from BM by IP (Figure 6A). The bacteriostatic effect of BM against $S$. aureus and GBS was significantly reduced after depletion of S100A8/A9 (Figure 6B). However, E. coli grew equally in unmodified and S100A8/A9-depleted BM (Figure 6B). Collectively, our data demonstrate that S100A8/A9 significantly contributes to the antimicrobial activity of BM against highly relevant, especially Mn-sensitive, pathogens of sepsis in newborns.

\section{DISCUSSION}

More than 400,000 babies die of neonatal sepsis per year worldwide making it one of the most common reasons for death in the neonatal period (9). Breast feeding reduces neonatal morbidity and mortality by infectious diseases as sepsis resulting in relevant cost savings to health-care providers (3, 10-12, 31-34). The molecular mechanisms how BM achieves these sepsis-protective effects are not yet completely elucidated. It is unquestionable that antimicrobial effects of BM contribute to the protection from infections and sepsis. Direct growth inhibition of $S$. aureus and E. coli by BM has been demonstrated, but the molecular mechanism remained unclear; bacteriostatic effects were only correlated with levels of antimicrobial proteins in $\mathrm{BM}$, correlating best with lactoferrin $(27,28)$. However, until now, most efforts of nutritional supplementation of formula with bioactive components from human BM remained without success in the clinical setting and did not decrease the incidence of sepsis $(2,13)$. Next to antimicrobial activity, immunoregulatory effects of BM might be even more relevant with respect to sepsis protection since neonates are primed to respond hyperinflammatory upon microbial challenges ending up with rapid fatal courses of sepsis $(6,14-16)$. However, mediators and molecular mechanisms of immunoregulation by BM are still broadly elusive.

In this study, we hypothesized that S100-alarmins might be good bioactive candidate components that unify immunoregulatory $(14-16)$ and antimicrobial $(18,19)$ properties of BM. For the first time, we showed that S100A8/A9 levels in BM after birth were at least six times higher than the already elevated S100A8/A9 serum levels in healthy term newborns. Similar to the postnatal course of S100A8/A9 serum levels of the descendants (14), S100A8/A9 concentrations in BM decreased over the first few weeks after birth to normal adult serum levels. They were 


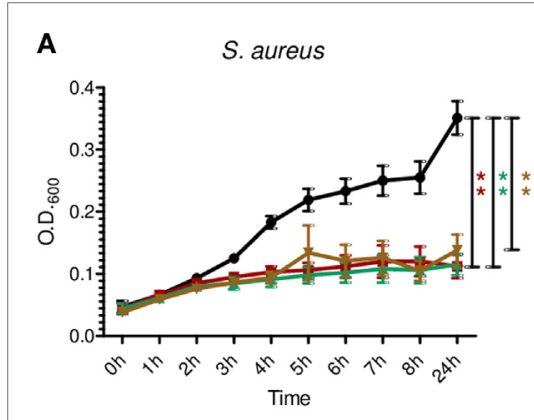

B

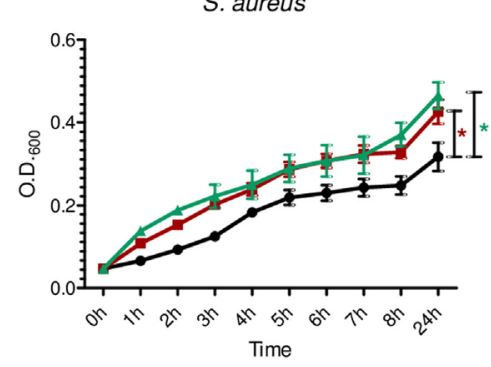

E. coli

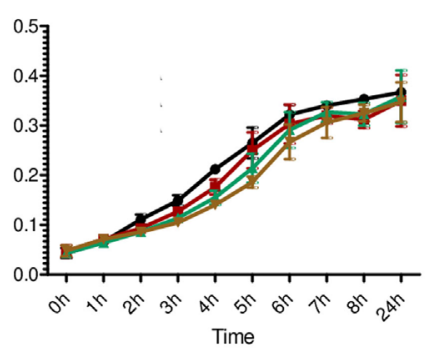

E. coli

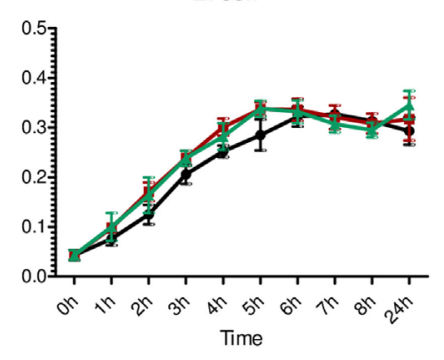

GBS

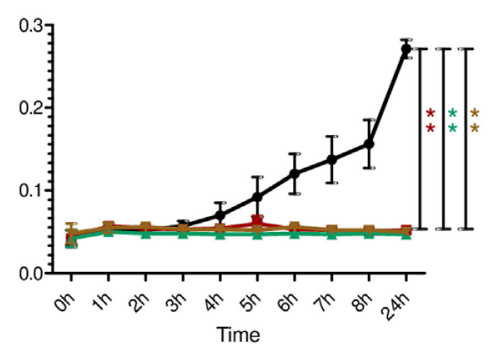

GBS

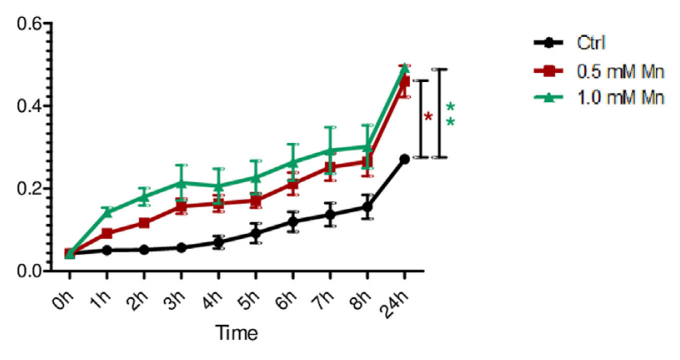

FIGURE 5 | Differential susceptibility of bacterial growth to metal ion depletion. Staphylococcus aureus, Escherichia coli, and group B streptococci (GBS) were grown at indicated concentrations of EDTA (A) and manganese (B). OD ${ }_{600}$ values over time were plotted as mean \pm SEM. ${ }^{*} p<0.05$ and ${ }^{* *} p<0.001, t$-test $(n=3)$.

highest after term compared with preterm delivery and after VD compared with CS, both again comparable to conditions of S100A8/A9 serum levels in babies born term versus preterm (15) or born per VD compared with CS (unpublished data). Our findings point to a closely stress-associated release of S100A8/A9 into BM during the process of birth. Whereas S100A8/A9 BM levels of mothers after term delivery were strictly dependent on the mode of delivery, this was only a trend after extremely or very preterm delivery and did not hold true for the group of mothers after moderate preterm birth. A good explanation for the latter might be the high proportion of secondary CS (31\%) in the moderate preterm group. Secondary CS implicates a varying degree of contractions and labor that might be comparable to labor during VD leading to a stress-associated release of S100A8/A9 into the BM.

With respect to antimicrobial properties, it has previously been shown that S100A8/A9 only acts bacteriostatic at high concentrations starting at $20 \mu \mathrm{g} / \mathrm{mL}(18,19)$. BM contains high amounts of $\mathrm{Ca}$. We could show that in the presence of $\mathrm{Ca}$ antimicrobial activity becomes significant at $10 \mu \mathrm{g} / \mathrm{mL}$, i.e., at average concentrations detectable in human BM. At such high concentrations, depletion of divalent metal ions by S100A8/ A9 was shown to become relevant hampering bacterial growth $(18,19,29)$. In line with others $(27,28)$, we demonstrated that BM inhibited the growth of $S$. aureus, E. coli, and GBS, three of the most common causal pathogens of sepsis in newborns (7, 35-39). In this study, we could clearly show that S100A8/ A9 in BM contributes significantly to the bacteriostatic activity of BM on S. aureus and GBS as depletion of S100A8/A9 from $\mathrm{BM}$ enhanced the growth of these strains. However, the growth of E. coli in BM remained unaffected after removal of S100A8/
A9. The reason is most likely the demonstrated independence of E. coli on the presence of $\mathrm{Mn}$ in contrast to the high Mn-sensitivity of $S$. aureus and GBS growth. BM contains only trace amounts of $\mathrm{Mn}$ (about $2 \mu \mathrm{M}$ ) compared with an excess of $\mathrm{Zn}$ and iron (0.1-0.3 mM). Considering the chelating capacities of the highest S100A8/A9 levels $(1-2 \mu \mathrm{M})$ detectable in BM, Mn depletion is certainly the most relevant antimicrobial mechanism of S100A8/ A9 in BM. Consequently, the growth inhibitory effect of BM against $E$. coli must rather be mediated by other antimicrobial factors than S100A8/A9 present in BM.

Interestingly, with respect to immunoregulatory effects, the homodimer S100A8 has been demonstrated to be more potent in neonates than the heterodimer complex of S100A8/A9 $(15,16)$. However, in contrast to S100A8/A9, S100A8 did not inhibit the growth of any of the tested pathogens of neonatal sepsis (S. aureus, E. coli, and GBS), which is most likely due to the lack of transitional binding sites for divalent metal ions (29). These data are in accordance with findings of others on the antimicrobial activity of S100-alarmins on S. aureus and K. pneumoniae $(18,19)$.

The high amount of S100-alarmins in BM next to their massive systemic release by the newborn itself suggests that the supply of newborns with S100-alarmins is of vital importance. We previously showed that the release of S100-alarmins is an essential mechanism in newborn infants that prevents excessive inflammation and fatal septic courses by inducing a state of inflammatory hyporesponsiveness (14-16). In this study, we demonstrated in murine $5100 a 9^{-/-}$neonates that enterally supplied S100-alarmins can even be detected systemically in the plasma of fed mice. Immunoregulation by $\mathrm{S} 100$-alarmins is already active at serum concentrations one log level lower than the S100A8/A9 levels in 


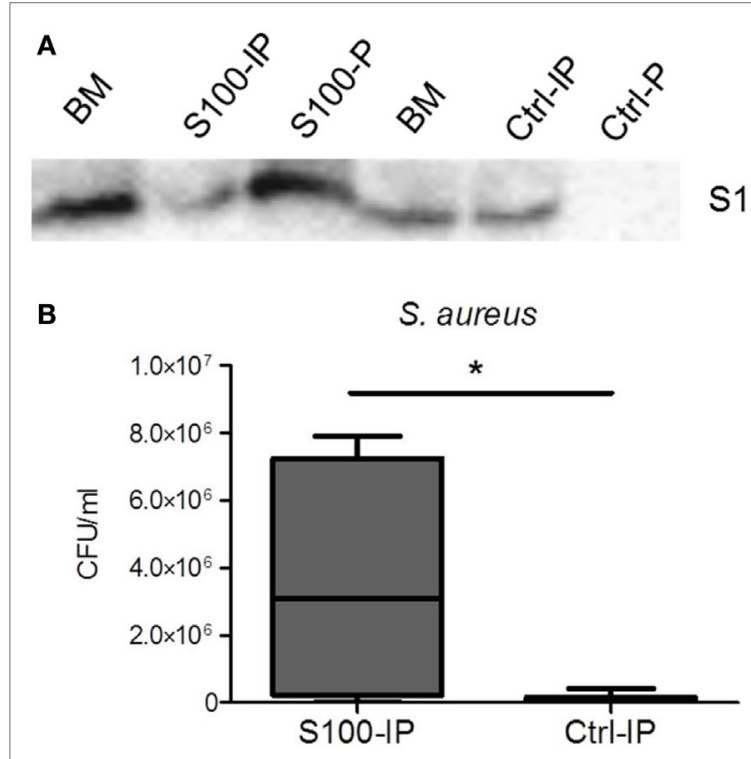

E. coli
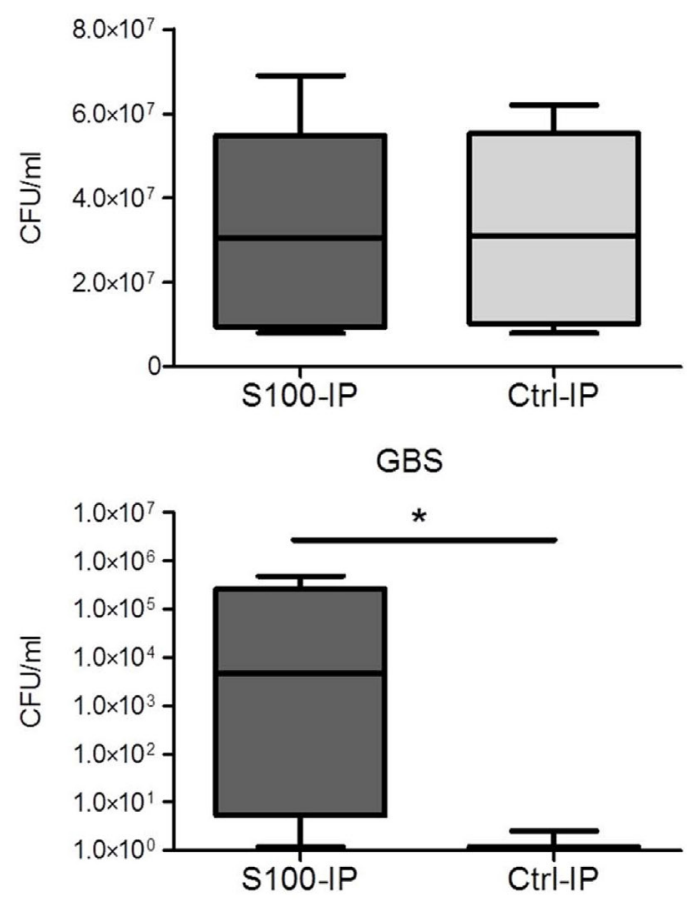

FIGURE 6 | Depletion of S100A8/A9 decreases the antimicrobial activity of breast milk (BM). (A) Efficiency of S100A8/A9 removal was determined by immunoblotting against S100A8. Representative blot showing BM probes before and after S100-IP and control (Ctrl)-IP as well as the precipitates (S100-P and Ctrl-P). (B) Staphylococcus aureus, Escherichia coli, and group B streptococci (GBS) were grown over 24 h in S100A8/A9-depleted (S100-IP) and non-depleted (Ctrl-IP) BM samples. Box plots show medians (center lines) and interquartile ranges of the concentration of colony-forming units $(\mathrm{CFU}) \pm$ SEM. ${ }^{*} p<0.05$, $t$-test.

BM. So, the biological relevance of S100A8/A9 in BM seems to be double. Due to their high concentrations in BM antimicrobial and immunoregulatory properties, both might contribute to the sepsis-protective effect of BM. Interestingly, Lee et al. demonstrated that early feeding with colostrum significantly reduces clinical sepsis in extremely low birth weight infants (40). We found $\mathrm{BM}$ of the first 2 days after delivery including colostrum to be massively loaded with $\mathrm{S} 100$-alarmins.

Next to the importance for the newborn infant, antimicrobially active amounts of S100A8/A9 in BM might also be important for the mother by protecting from lactational mastitis, especially given the fact that Staphylococci and Streptococci are the most common pathogens found in mastitis $(41,42)$.

Our investigations demonstrate that BM contains vast amounts of S100-alarmins. Levels are highest in the group of newborns with the lowest sepsis risk, namely, term infants delivered vaginally. Concentrations are so high that next to immunoregulatory functions S100A8/A9 in BM acts antimicrobially against important pathogens causing neonatal sepsis. These findings point to S100-alarmins as attractive bioactive components whose enteral supply could prevent fatal courses in newborns that are at high risk to suffer from sepsis and inflammation.

\section{ETHICS STATEMENT}

The studies were approved by the Institutional Review Board of Hannover Medical School (no. 6143-2012). Written informed consent was obtained from all participating women. Mouse experiments were in accordance with German Animal Welfare Legislation and performed as approved by the Lower Saxony State Office for Consumer and Food Safety, Germany (approval no. 33.12-42502-04-14 and no. 33.12-42502-04-15).

\section{AUTHOR CONTRIBUTIONS}

SP and DV conceptualized and designed the study; SP, MR, JP, and $\mathrm{CH}$ collected samples and data; $\mathrm{MR}, \mathrm{BF}$, and TV performed experiments; SP, CH, JR, TV, and DV analyzed the data; SP and MR drafted the initial manuscript; and SP, MR, CH, JR, TV, and DV reviewed and revised the manuscript. All the authors approved the final manuscript as submitted and agreed to be accountable for all aspects of the work.

\section{ACKNOWLEDGMENTS}

The authors are grateful to all mothers contributing to this study. They thank Dr. Lena Völlger for critically reading the manuscript.

\section{FUNDING}

This work was supported by grants from the Appenrodt Foundation, the German Research Foundation [VI 538/6-1]; and the Volkswagen Foundation [Az 90005] to DV; from the Interdisciplinary Center of Clinical Research at the University of Münster [Vo2/014/09, Ro2/003/15], the cluster of excellence Cells in Motion and the collaborative research centers 1009 [B08, B09]; and the German Research Foundation [TRR34] to $\mathrm{TV}$ and JR, and by a clinical leave stipend of the German Center of Infection Research (DZIF) and a junior grant of the University of Lübeck to JP. 


\section{REFERENCES}

1. American Academy of Pediatrics. Policy statement. Section on breastfeeding. Breastfeeding and the use of human milk. Pediatrics (2012) 129:e827e41. doi:10.1542/peds.2011-3552

2. Lönnerdal B. Bioactive proteins in human milk: health, nutrition, and implications for infant formulas. J Pediatr (2016) 173S:S4-9. doi:10.1016/j. jpeds.2016.02.070

3. Sankar MJ, Sinha B, Chowdhury R, Bhandari N, Taneja S, Martines J, et al. Optimal breastfeeding practices and infant and child mortality: a systematic review and meta-analysis. Acta Paediatr (2015) 104(467):3-13. doi:10.1111/ apa.13147

4. Labbok MH, Clark D, Goldman AS. Breastfeeding: maintaining an irreplaceable immunological resource. Nat Rev Immunol (2004) 4(7):565-72. doi: $10.1038 /$ nril393

5. Underwood MA. Missed opportunities: the cost of suboptimal breast milk feeding in the neonatal intensive care unit. J Pediatr (2016) 175:12-4. doi:10.1016/j.jpeds.2016.05.016

6. Zhao J, Kim KD, Yang X, Auh S, Fu YX, Tang H. Hyper innate responses in neonates lead to increased morbidity and mortality after infection. Proc Natl Acad Sci U S A (2008) 105:7528-33. doi:10.1073/pnas.0800152105

7. Shane AL, Stoll BJ. Neonatal sepsis: progress towards improved outcomes. J Infect (2014) 68(Suppl 1):S24-32. doi:10.1016/j.jinf.2013.09.011

8. Weiss SL, Fitzgerald JC, Pappachan J, Wheeler D, Jaramillo-Bustamante JC, Salloo A, et al. Global epidemiology of pediatric severe sepsis: the sepsis prevalence, outcomes, and therapies study. Am J Respir Crit Care Med (2015) 191(10):1147-57. doi:10.1164/rccm.201412-2323OC

9. Liu L, Oza S, Hogan D, Perin J, Rudan I, Lawn JE, et al. Global, regional, and national causes of child mortality in 2000-13, with projections to inform post-2015 priorities: an updated systematic analysis. Lancet (2015) 385(9966):430-40. doi:10.1016/S0140-6736(14)61698-6

10. Colaizy TT, Bartick MC, Jegier BJ, Green BD, Reinhold AG, Schaefer AJ, et al. Impact of optimized breastfeeding on the costs of necrotizing enterocolitis in extremely low birthweight infants. J Pediatr (2016) 175:100-5. doi:10.1016/j. jpeds.2016.03.040

11. Schanler RJ, Lau C, Hurst NM, Smith EO. Randomized trial of donor human milk versus preterm formula as substitutes for mothers' own milk in the feeding of extremely premature infants. Pediatrics (2005) 116(2):400-6. doi:10.1542/peds.2004-1974

12. Schanler RJ, Shulman RF, Lau C. Feeding strategies for premature infants: beneficial outcomes of feeding fortified human milk versus preterm formula. Pediatrics (1999) 103(6 Pt 1):1150-7. doi:10.1542/peds.103.6.1150

13. Yu W, Sui W, Mu L, Yi W, Li H, Wei L, et al. Preventing necrotizing enterocolitis by food additives in neonates. A network meta-analysis revealing the efficacy and safety. Medicine (2017) 96(21):e6652. doi:10.1097/ MD.0000000000006652

14. Austermann J, Friesenhagen J, Fassl SK, Petersen B, Ortkras T, Burgmann J, et al. Alarmins MRP8 and MRP14 induce stress tolerance in phagocytes under sterile inflammatory conditions. Cell Rep (2014) 9:2112-23. doi:10.1016/j. celrep.2014.11.020

15. Ulas T, Pirr S, Fehlhaber B, Bickes MS, Loof TG, Vogl T, et al. S100-induced innate immune programming protects newborn infants from sepsis. Nat Immunol (2017) 18(6):622-32. doi:10.1038/ni.3745

16. Heinemann AS, Pirr S, Fehlhaber B, Mellinger L, Burgmann J, Busse M, et al. In neonates S100A8/S100A9 alarmins prevent the expansion of a specific inflammatory monocyte population promoting septic shock. FASEB J (2017) 31:1153-64. doi:10.1096/fj.201601083R

17. Vogl T, Tenbrock K, Ludwig S, Leukert N, Ehrhardt C, van Zoelen MA, et al. Mrp8 and Mrp14 are endogenous activators of toll-like receptor 4, promoting lethal, endotoxin-induced shock. Nat Med (2007) 13:1042-9. doi:10.1038/ nm1638

18. Corbin BD, Seeley EH, Raab A, Feldmann J, Miller MR, Torres VJ, et al. Metal chelation and inhibition of bacterial growth in tissue abscesses. Science (2008) 319:962-5. doi:10.1126/science.1152449

19. Achouiti A, Vogl T, Urban CF, Röhm M, Hommes TJ, van Zoelen MA, et al. Myeloid-related protein-14 contributes to protective immunity in gramnegative pneumonia derived sepsis. PLoS Pathog (2012) 8(10):e1002987. doi:10.1371/journal.ppat.1002987
20. Cho H, Jeong DW, Liu Q, Yeo WS, Vogl T, Skaar EP, et al. Calprotectin increases the activity of the SaeRS two component system and murine mortality during Staphylococcus aureus infections. PLoS Pathog (2015) 11(7):e1005026. doi:10.1371/journal.ppat.1005026

21. Achouiti A, Vogl T, Endeman H, Mortensen BL, Laterre PF, Wittebole X, et al. Myeloid-related protein-8/14 facilitates bacterial growth during pneumococcal pneumonia. Thorax (2014) 69:1034-42. doi:10.1136/ thoraxjnl-2014-205668

22. van den Bos C, Rammes A, Vogl T, Boynton R, Zaia J, Sorg C, et al. Copurification of P6, MRP8, and MRP14 from human granulocytes and separation of individual proteins. Protein Expr Purif (1998) 13(3):313-8. doi:10.1006/prep.1998.0917

23. Vogl T, Leukert N, Barczyk K, Strupat K, Roth J. Biophysical characterization of S100A8 and S100A9 in the absence and presence of bivalent cations. Biochim Biophys Acta (2006) 1763(11):1298-306. doi:10.1016/j. bbamcr.2006.08.028

24. Frosch M, Strey A, Vogl T, Wulffraat NM, Kuis W, Sunderkötter C, et al. Myeloid-related proteins 8 and 14 are specifically secreted during interaction of phagocytes and activated endothelium and are useful markers for monitoring disease activity in pauciarticular-onset juvenile rheumatoid arthritis. Arthritis Rheum(2000)43(3):628-37.doi:10.1002/1529-0131(200003)43:3<628:AIDANR20>3.0.CO;2-X

25. Manitz MP, Horst B, Seeliger S, Strey A, Skryabin BV, Gunzer M, et al. Loss of S100A9 (MRP14) results in reduced interleukin-8-induced CD11b surface expression, a polarized microfilament system, and diminished responsiveness to chemoattractants in vitro. Mol Cell Biol (2003) 23(3):1034-43. doi:10.1128/ MCB.23.3.1034-1043.2003

26. Viemann D, Goebeler M, Schmid S, Klimmek K, Sorg C, Ludwig S, et al. Transcriptional profiling of IKK2/NF-kappa B- and p38 MAP kinasedependent gene expression in TNF-alpha-stimulated primary human endothelial cells. Blood (2004) 103:3365-73. doi:10.1182/blood-2003-09-3296

27. Trend S, Strunk T, Hibbert J, Kok CH, Zhang G, Doherty DA, et al. Antimicrobial protein and peptide concentrations and activity in human breast milk consumed by preterm infants at risk of late-onset neonatal sepsis. PLoS One (2015) 10(2):e0117038. doi:10.1371/journal.pone.0117038

28. van Gysel M, Cossey V, Fieuws S, Schuermans A. Impact of pasteurization on the antibacterial properties of human milk. Eur J Pediatr (2012) 171:1231-7. doi:10.1007/s00431-012-1750-4

29. Kehl-Fie TE, Chitayat S, Hood MI, Damo S, Restrepo N, Garcia C, et al. Nutrient metal sequestration by calprotectin inhibits bacterial superoxide defense enhancing neutrophil killing of Staphylococcus aureus. Cell Host Microbe (2011) 10(2):158-64. doi:10.1016/j.chom.2011.07.004

30. Nakashige TG, Zhang B, Krebs C, Nolan EM. Human calprotectin is an iron-sequestering host-defense protein. Nat Chem Biol (2015) 11(10):765-71. doi:10.1038/nchembio.1891

31. Corpeleijn WE, Kouwenhoven SMP, Paap MC, van Vliet I, Scheerder I, Muizer Y, et al. Intake of own mother's milk during the first days of life is associated with decreased morbidity and mortality in very low birth weight infants during the first 60 days of life. Neonatology (2012) 102:276-81. doi:10.1159/000341335

32. Victora CG, Bahl R, Barros AJD, França GV, Horton S, Krasevec J, et al. Breastfeeding in the 21st century: epidemiology, mechanisms, and lifelong effect. Lancet (2016) 387:475-90. doi:10.1016/S0140-6736(15)01024-7

33. Mahon J, Claxton L, Wood H. Modelling the cost-effectiveness of human milk and breastfeeding in preterm infants in the United Kingdom. Health Econ $\operatorname{Rev}(2016)$ 6:54-61. doi:10.1186/s13561-016-0136-0

34. Patel AL, Johnson TJ, Engstrom JL, Fogg LF, Jegier BJ, Bigger HR, et al. Impact of early human milk on sepsis and health-care costs in very low birth weight infants. J Perinatol (2013) 33:514-9. doi:10.1038/jp.2013.2

35. Stoll BJ, Hansen NI, Sánchez PJ, Faix RG, Poindexter BB, Van Meurs KP, et al. Eunice Kennedy Shriver National Institute of Child Health and Human Development Neonatal Research Network. Early onset neonatal sepsis: the burden of group B streptococcal and E. coli disease continues. Pediatrics (2011) 127:817-26. doi:10.1542/peds.2010-2217

36. Vergnano S, Menson E, Kennea N, Embleton N, Russell AB, Watts T, et al. Neonatal infections in England: the NeonIN surveillance network. Arch Dis Child Fetal Neonatal Ed (2011) 96:F9-14. doi:10.1136/adc.2009. 178798 
37. Tröger B, Härtel C, Buer J, Dördelmann M, Felderhoff-Müser U, Höhn T, et al. Clinical relevance of pathogens detected by multiplex PCR in blood of very-low-birth weight infants with suspected sepsis - Multicentre Study of the German Neonatal Network. PLoS One (2016) 11(7):e0159821. doi:10.1371/ journal.pone.0159821

38. Dong Y, Speer CP. Late-onset neonatal sepsis: recent developments. Arch Dis Child Fetal Neonatal Ed (2015) 100:F257-63. doi:10.1136/archdischild2014-306213

39. Bizzarro MJ, Shabanova V, Baltimore RS, Dembry LM, Ehrenkranz RA, Gallagher PG. Neonatal sepsis 2004-2013: the rise and fall of coagulasenegative staphylococci. J Pediatr (2015) 166(5):1193-9. doi:10.1016/j.jpeds. 2015.02.009

40. Lee J, Kim HS, Jung YH, Choi KY, Shin SH, Kim EK, et al. Oropharyngeal colostrum administration in extremely premature infants: an RCT. Pediatrics (2015) 135(2):e357-66. doi:10.1542/peds.2014-2004

41. Mediano P, Fernández L, Jiménez E, Arroyo R, Espinosa-Martos I, Rodríguez JM, et al. Microbial diversity in milk of women with mastitis: potential role of coagulase-negative staphylococci, viridans group streptococci, and corynebacteria. J Hum Lact (2017) 33(2):309-18. doi:10.1177/0890334417692968

42. Kvist LJ, Larsson BW, Hall-Lord ML, Stehen A, Schalén C. The role of bacteria in lactational mastitis and some considerations of the use of antibiotic treatment. Int Breastfeed J (2008) 3:6. doi:10.1186/1746-4358-3-6

Conflict of Interest Statement: The authors declare that the research was conducted in the absence of any commercial or financial relationships that could be construed as a potential conflict of interest.

Copyright $\odot 2017$ Pirr, Richter, Fehlhaber, Pagel, Härtel, Roth, Vogl and Viemann. This is an open-access article distributed under the terms of the Creative Commons Attribution License (CC BY). The use, distribution or reproduction in other forums is permitted, provided the original author(s) or licensor are credited and that the original publication in this journal is cited, in accordance with accepted academic practice. No use, distribution or reproduction is permitted which does not comply with these terms. 